http://jmscr.igmpublication.org/home/ ISSN (e)-2347-176x ISSN (p) 2455-0450

crossref DOI: https://dx.doi.org/10.18535/jmscr/v8i2.159

\author{
Journal Of Medical Science And Clinical Research \\ IGM Publication \\ An official Publication of IGM Publication
}

\title{
Skin Prick Test in Chronic Urticaria in a Tertiary Care Centre in South India
}

\section{Authors \\ Sreejith. A.P ${ }^{1}$, Anuja Elizabeth George ${ }^{2}$, Rony Mathew ${ }^{3}$}

${ }^{1}$ Department of Dermatology and Venereology, Government Medical College, Thiruvananthapuram, Kerala, India ${ }^{2}$ Department of Dermatology and Venereology, Government Medical College, Thiruvananthapuram, Kerala, India ${ }^{3}$ Department of Dermatology and Venereology, Government T.D. Medical College, Alappuzha, Kerala, India

* Corresponding Author

Dr Anuja Elizabeth George

Professor \& Head of Department, Department of Dermatology \& Venereology,

Government Medical College, Thiruvananthapuram, Kerala, India 695011

\begin{abstract}
Background: Urticaria is a cutaneous disease with short-lived itchy wheals, angio-oedema or both. Recurrence of lesions for 6 weeks or more is considered as chronic. Chronic urticaria is estimated to have a life time prevalence of $1.8 \%$. The peak incidence of chronic spontaneous urticaria is in the fourth to fifth decade and seriously compromises the quality of life of patients due to debilitating and uncomfortable symptoms that may last for years. Skin prick test (SPT) is a reliable, convenient and less expensive method to detect IgE mediated allergy and helps to identify the allergens to which patients with chronic urticaria are sensitised.

Aims and Objectives: To determine the occurrence of positive skin prick test in chronic urticaria and to identify the allergens implicated in them.

Methods: Seventy seven patients above 12 years of age, attending the Department of Dermatology and Venereology for chronic urticaria were included as study subjects. They were given SPT with 52 purified extracts of allergens commonly seen in this region.

Results: SPT was found to be positive in 53 patients (68.8\%). Highest sensitization was seen to insect allergens (45.5\%), followed by food (20.8\%), house dust mite (18\%), pollen (13\%), fungi (5.2\%), house dust $(2.6 \%)$ and dander (2.6\%).

Conclusion: This study showed that $68.8 \%$ of patients with chronic urticaria were sensitised to different types of allergens found locally in this region. Skin prick test can be helpful in determining probable causative allergens and thereby aid in managing this chronic condition.

Keywords: Chronic urticaria, skin prick test, allergens.
\end{abstract}

\section{Introduction}

Urticaria presents with short-lived itchy wheals, angio-oedema or both. ${ }^{[1]}$ Classification of urticaria is based on both duration as well as the causes or triggers of urticaria. Acute urticaria is defined as repeated appearance of wheals, with or without angioedema, lasting less than 6 weeks. Recurrence of lesions for 6 weeks or more is considered as chronic. ${ }^{[2]}$ Chronic urticaria can be either chronic spontaneous urticaria to known or unknown causes $^{[2]}$ or inducible urticaria where specific stimulus induces reproducible wheals. About 30- 
$50 \%$ patients with chronic spontaneous urticaria have circulating auto-antibodies and are labelled as autoimmune urticaria while about $50 \%$ of cases have no provoking factors or circulating antibodies and they come under chronic idiopathic urticaria. The lifetime prevalence of chronic urticaria is $1.8 \%{ }^{[3]}$ Worldwide incidence is $0.1 \%-3 \%$ of population with women affected twice more than men. ${ }^{[4]}$ The peak incidence is in the fourth and fifth decade and can cause debilitating symptoms which may last for years and affect the quality of life of patients.

Immediate hypersensitivity is thought to play a role in the pathogenesis of chronic urticaria. SPT is a reliable method to detect presence of allergen specific IgE on patient's mast cells and thus diagnose $\operatorname{IgE}$ mediated allergic disease. Identification of the probable causative allergen and its avoidance will aid in controlling chronic urticaria. This study was undertaken to estimate the positive SPT in patients with chronic urticaria and to identify the various allergens implicated in them.

\section{Materials and Methods}

Seventy seven patients above the age of 12 years, who presented with symptoms of chronic spontaneous urticaria during a period of one year were included in this hospital based cross sectional study. Patients with acute symptoms of allergy; severe dermographism; pregnant, those on beta blockers and ACE inhibitors; those unable to stop antihistamines, steroids etc prior to test and those with history of anaphylaxis were excluded. A written informed consent was obtained from all the participants and confidentiality of records was maintained.

The patients were instructed to stop the intake of the following medicines prior to the test as per the schedule: Corticosteroids: 2weeks prior; First generation antihistamines: 3 days prior; Second generation antihistamines: 1week prior; Tricyclic antidepressants and mast cell stabilisers: 1week prior to the test.

Structured questionnaire was used for data collection. Clinical and demographic details as well as history of any factors precipitating urticaria were noted and laboratory investigations relevant to urticaria were done. Lab investigations included routine blood investigations, routine urine examination, liver function tests, renal function test, blood VDRL \& TPHA, Thyroid function test, Hepatitis B surface antigen, antibodies to Hepatitis $\mathrm{C}$ virus, Antinuclear antibody test, IgG H-pylori, Anti Streptolysin O and Mantoux test.

Skin prick test was done in these patients with 52 purified extracts of allergens commonly seen in this region.

\section{Procedure for Skin prick test}

The test site chosen is the volar aspect of forearm, $2 \mathrm{~cm}$ from the wrist and the cubital fossa. A positive control is done using Histamine phosphate solution and negative control using buffered saline in glycerol base. Positive and negative controls are applied and the time is noted. Adjacent test sites are marked $2 \mathrm{~cm}$ apart on the volar aspect of both forearms. Droplets of allergens are applied on the test site and the skin is pricked through the droplet with a lancet, $1 \mathrm{~mm}$ deep to pierce the epidermis. Only one antigen is applied and pricked at a time. Care is taken to avoid mixing of adjacent antigens. Excess antigens are blotted off with blotting paper. Any wheal formed is measured after 15-20 minutes with a standard ruler for the greatest diameter. Pseudo-podial extensions are discarded. Wheal diameter $\geq 3 \mathrm{~mm}$ of negative control is considered as positive. The data were entered in excel and analysed by EPI NFO 7 software.

\section{Results}

Among the 77 patients with chronic urticaria, there were 27 males (35.1\%) and 50 females (64.9\%). The male to female ratio was $1: 1.85$.

The age of patients ranged from 13-65 years. The mean age was $36.33 \pm 13.28$ years. There were 23 (29.9\%) patients in the age group 33-42 years, followed by $20(26 \%)$ in 23-32 age group and 13 (16.9\%) patients in 43-52 the age group. Twelve $(15.5 \%)$ patients were between 13 and 22 years, 5 $(6.5 \%)$ between 53 and 62 years and $4(5.2 \%)$ were $\geq 62$ years. (Figure: 1 ) 
Duration of the disease ranged from 2 months to 13 years (mean $18.07 \pm 12.09$ months). Maximum number of patients, 34 (44.2\%) had chronic urticaria for $<1$ year while 22 patients $(28.5 \%)$ had it for $>2$ years. Patients having urticaria for 1-2 years were $21(27.3 \%)$.

Aggravating factors were present in 37 patients (48.1\%). Food was reported as the aggravating factor in 25 patients $(32.5 \%)$; followed by pressure in $8(10.4 \%)$; exercise in $5(6.5 \%)$; sunlight in 4 $(5.2 \%)$ and cold in 1 patient $(1.3 \%)$.

History of intermittent and mild angioedema was reported in $26(33.8 \%)$ patients, while there was no such history of angioedema in $51(66.2 \%)$ patients.

History of atopy was reported in $9(11.7 \%)$ patients only, among whom, 3 patients had bronchial asthma, 4 had history of allergic rhinitis and 2 had atopic dermatitis. Mild dermographism was noted in 9 patients $(11.7 \%)$.

Among the 77 patients with chronic urticaria, SPT was positive in $53(68.8 \%)$. Positive SPT is shown in Figure:2. Among them, 17 (22.1\%) patients were positive to only one allergen, $13(16.8 \%)$ were positive to 2 allergens and $12(15.6 \%)$ to 3 allergens. There were $7(9.1 \%)$ patients who were positive for 4 allergens, $3(3.9 \%)$ patients to five allergens and one $(1.3 \%)$ patient was positive to seven allergens.

The number of patients who were positive for each allergen tested is shown in Table: 1 . Out of the total 77 patients, insect allergens produced maximum sensitisation with 35 patients $(45.5 \%)$ and some persons being SPT positive to multiple antigens. Most common insect allergens were housefly and male cockroach, 18 each $(23.3 \%)$.

Food allergens were positive in 16 patients (20.8\%), some being SPT positive to multiple antigens. The most common food allergens were wheat, chicken and prawn, 3 each (3.9\%).

Sensitivity to house dust mite was seen in 14 (18.2\%) patients and to pollen in $10(13 \%)$ patients.
The most common pollen allergen showing SPT positivity was Lawsonia inermis (Henna) in 5 (6.5\%) patients, followed by Carica papaya and Parthenium, in $2(2.6 \%)$ patients each. Four patients $(5.2 \%)$ were found sensitised to different types of fungal allergens, the most common being Aspergillus and Rhizopus, in 2 (2.6\%) each. House dust and animal dander produced sensitivity in 2 patients $(2.6 \%)$ each, one patient each positive for dog and cat dander. There was no SPT positivity to fabric.

Only 9 patients $(11.7 \%)$ had history of atopy, and 6 of them $(66.7 \%)$ showed positive SPT. Among these 9 patients, $5(55.5 \%)$ were sensitive to insects, $3(33.3 \%)$ to house dust mite and $2(22.2 \%)$ to food.

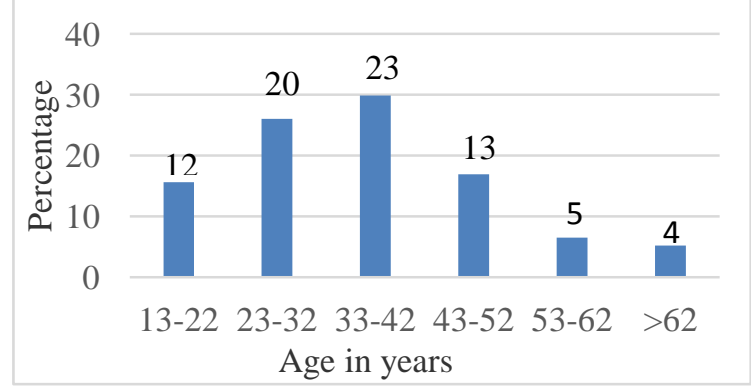

Figure 1: Age

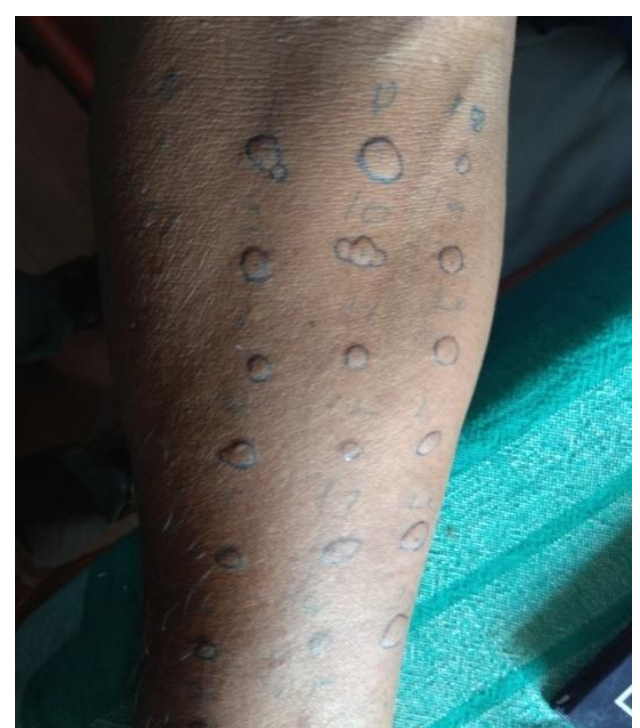

Figure 2: Positive skin prick test 
Table 1: Result of skin prick test to specific allergens

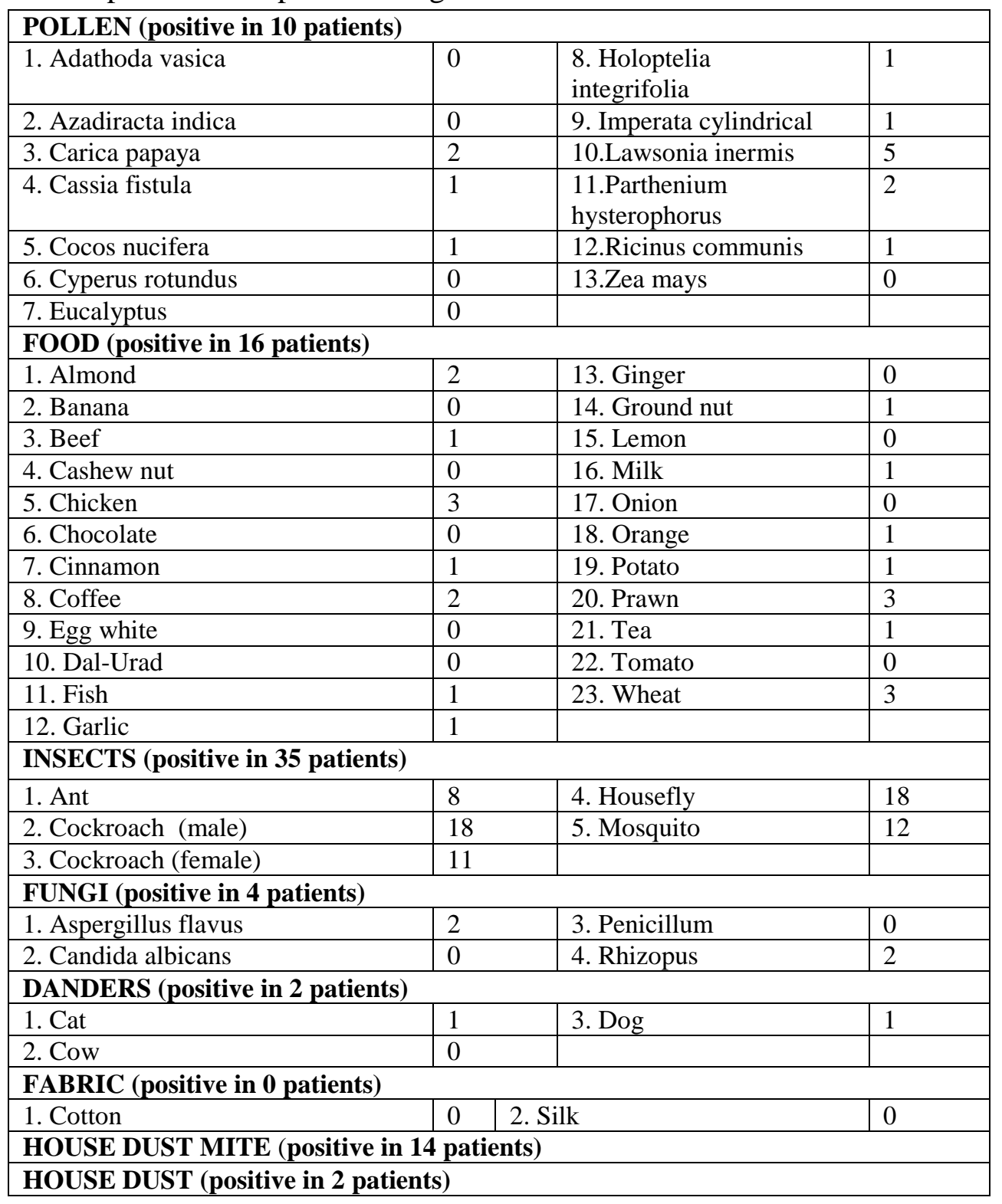

Table 2: Comparison of SPT positivity to allergens in various studies

\begin{tabular}{|l|c|c|c|c|c|c|c|c|}
\hline Studies & No of patients & Insects & Food & $\begin{array}{c}\text { House } \\
\text { dust mite }\end{array}$ & Pollen & Fungi & $\begin{array}{c}\text { House } \\
\text { dust }\end{array}$ & Dander \\
\hline Nath et al & 50 & $24 \%$ & $22 \%$ & $12 \%$ & $24 \%$ & $10 \%$ & & $14 \%$ \\
\hline Pooja Bains & 41 & $17 \%$ & $21.9 \%$ & $7.32 \%$ & $26.83 \%$ & & $26.83 \%$ & 0 \\
\hline Rebello MS & 40 & $32 \%$ & $28 \%$ & $50 \%$ & $22 \%$ & & & \\
\hline Kulthanan et al & 88 & & $30 \%$ & & & & & \\
\hline This study & $\mathbf{7 7}$ & $\mathbf{4 5 . 5 \%}$ & $\mathbf{2 0 . 8 \%}$ & $\mathbf{1 8 \%}$ & $\mathbf{1 3 \%}$ & $\mathbf{5 . 2 \%}$ & $\mathbf{2 . 6 \%}$ & $\mathbf{2 . 6 \%}$ \\
\hline
\end{tabular}

\section{Discussion}

Skin prick test done on 77 patients with chronic urticaria, during a period of one year was positive in $53(68.8 \%)$ patients, which was comparable with the study done by Bains P et al (63.4\%). ${ }^{[5]}$

Females outnumbered (50, 64.9\%) males (27, $35.1 \%$ ) similar to the observation by Khan et al and Kulthanan $(86.4 \%){ }^{[4],[6]}$
In this study, sensitivity was maximum to insects (35, 45.5\%); followed by food $(16,20.8 \%)$; house dust mite $(14,18 \%)$; pollen $(10,13 \%)$; fungi (4, $5.2 \%)$, house dust $(2,2.6 \%)$; dander $(2,2.6 \%)$ and there was no sensitivity to fabric allergen. Table: 2 shows the comparison with other studies.

In this study, 17 patients showed positive reaction to only a single allergen (22.1\%), while 36 of them 
were positive for 2 or more allergens. The maximum number of allergens positive in a single patient was 7, and was seen in only one patient. Rebello M S et al obtained 1-5 allergen positivity in 13 out of 40 patients (32.5\%), 6-10 allergen positivity in 15 patients $(37.5 \%)$ and more than 10 allergen positivity in 5 patients $(12.5 \%){ }^{[7]}$

The study by Nath et al, was comparable with this study, although the prevalence was lesser, as also the number of study subjects. Out of 50 patients with chronic urticaria, the maximum positive reactions were seen to insects and pollen, 12 patients each (24\%), followed by food in 11 patients (22\%), animal dander in 7 patients (14\%) and house dust mite in 6 patients $(12 \%) .{ }^{[8]}$

Insect allergen which showed maximum positivity were 18 each of housefly and male cockroach (23.3\%) followed by mosquito in $12(15.5 \%)$. Bains $\mathrm{P}$ et al reported cockroaches, grasshopper and rice weevil $(4.9 \%$ each) as most common insect allergens in their study. ${ }^{[5]}$

Most common food allergens were wheat, chicken and prawn in 3 each (3.9\%). Bains $\mathrm{P}$ et al found chicken, mushroom and coffee as the most common food allergens (4.8\% each) while Rebello MS et al found prawn $(3.2 \%)$, chicken $(2.7 \%)$ and wheat $(2 \%){ }^{[7]}$

Lawsonia inermis (Henna) was the most common pollen allergen in this study $(5,6.5 \%)$, followed by $2(2.6 \%)$ each of Parthenium and Carica papaya. Nath et al reported $4 \%$ of patients sensitive to Parthenium and Bains $\mathrm{P}$ et al reported $4.88 \%$ Parthenium sensitivity. ${ }^{[8],[5]}$

The most common fungal allergens which showed positive reactions were Aspergillus and Rhizopus, 2 each $(2.6 \%)$. Nath et al reported $2 \%$ of patients positive to Aspergillus. ${ }^{[8]}$

There was one patient each $(1.3 \%)$ sensitive to cat and dog dander in this study. Nath et al reported $12 \%$ and $2 \%$ positivity respectively to these allergens. $^{[8]}$

History of atopy was present in only 9 patients (11.7\%), of which 6 patients $(66.7 \%)$ had positive SPT. Sensitivity to insects was seen in 5 atopic patients $(55.5 \%), 3$ patients $(33.3 \%)$ were sensitive to house dust mite and $2(22.2 \%)$ to food. Kulthanan et al observed house dust mite sensitivity in $66.1 \%$ of the patients with atopy and Mahesh et al reported $79 \%$ patients with atopy as house dust mite sensitive. ${ }^{[9],[10]}$

\section{Conclusion}

Chronic urticaria is a disabling condition with a life time prevalence of $1.8 \%$, and affecting the quality of life of patients. In this study, $68.8 \%$ of patients with chronic urticaria were found to be sensitised to different types of common allergens found in this region. Finding and eliminating the precipitating cause can be helpful in managing chronic urticaria. Sensitivity to various allergens in patients with chronic urticaria may be detected by skin prick test. Skin prick test, being a simple and inexpensive tool can be helpful in determining these allergens and aid in managing this chronic condition by avoiding exposure to these allergens.

\section{References}

1. Zuberbier T. Classification of urticaria. Indian J Dermatol. 2013;58:208

2. Zuberbier T, Aberer W, Asero R, BindslevJensen C, Brzoza Z, Canonica GW, et al. The EAACI/GA 2 LEN/EDF/WAO Guideline for the definition, classification, diagnosis, and management of urticaria: the 2013 revision and update. Allergy. 2014;69:86887.

3. Zuberbier T, Balke M, Worm M, Edenharter G, Maurer M. Epidemiology of urticaria: a representative cross-sectional population survey: Epidemiology of urticaria: a representative cross-sectional population survey. Clin Exp Dermatol. 2010;35:869-73.

4. Khan S, Maitra A, Hissaria P, Roy S, Padukudru Anand M, Nag N, et al. Chronic Urticaria: Indian Context-Challenges and Treatment Options. Dermatol Res Pract. 2013;2013:1-8.

5. Bains P, Dogra A. Skin prick test in patients with chronic allergic skin disorders. Indian $\mathrm{J}$ Dermatol. 2015;60:159-64. 
6. Kulthanan $\mathrm{K}$, Jiamton $\mathrm{S}$, Rutnin $\mathrm{N}$, Insawang M, Pinkaew S. Prevalence and relevance of positivity of skin prick testing in patients with chronic urticaria. J Dermatol. 2008;35:330-5.

7. Sonia Rebello M, Bhatt R, D S, Alapatt GF. A study of skin prick in patients with chronic urticaria. International Journal of Recent Trends in Science and Technology. 17:14-7.

8. Nath A, Balaji A, Thappa DM. Prick testing in chronic idiopathic urticaria: A report from a tertiary care centre in south India.Internat J Dermatol.2007;6.

9. Kulthanan K, Chularojanamontri L, Manapajon A, Nuchkull P. Prevalence and clinical characteristics of adult-onset atopic dermatitis with positive skin prick testing to mites. Asian Pac J Allergy Immunol.2011;29:318.

10. Mahesh P, Kushalappa P, Holla A, Vedanthan P. House dust mite sensitivity is a factor in chronic urticaria. Indian $\mathbf{J}$ Dermatol Venereol Leprol.2005;71:99. 